\title{
SYMPTOMATIC OSSICLES OF THE LATERAL MALLEOLUS IN CHILDREN
}

\author{
JOHN D. GRIFFITHS, MALCOLM B. MENELAUS \\ From the Royal Children's Hospital, Melbourne
}

\begin{abstract}
We describe three children with symptoms of damage to the attachment of an anomalous ossific centre of the lower fibular epiphysis. All three were aged 8 to 10 years at the time of the initial injury, had suffered recurrent ankle sprains and had well localised and consistent tenderness precisely at the site of the anomalous ossific centre. All their symptoms were relieved by excision of the ossicle with reconstitution of the fibular collateral ligament. Whilst a separate secondary centre of ossification at the lower fibula is present in $1 \%$ of healthy children between the ages of 6 and 12 years, the condition described is extremely uncommon. Excision of the fragment should be reserved for those patients with recalcitrant symptoms and with consistent tenderness precisely at the site of the accessory ossicle.
\end{abstract}

Accessory ossicles are small, well-corticated supernumerary bones commonly found in the foot and ankle. Some $22 \%$ of normal children under the age of 16 have one or more accessory ossicles in the foot and ankle (Shands 1931); these are usually identified when radiographs are taken after an injury to the ankle. Both diagnostic and medico-legal problems may arise (Watkins 1937), since an accessory ossicle may represent anomalous ossification, a sesamoid bone or an avulsion fracture. This article is concerned with the first of these types of ossicle.

Normally, the secondary centre of ossification of the lateral malleolus appears during the first year of life, and fuses with the shaft between the ages of 15 and 17 years (Francis and Werle 1939). In a study of healthy children between 6 and 12 years old, Powell (1961) found a separate centre of ossification for the medial malleolus in $20 \%$ of cases, and for the lateral malleolus in $1 \%$; these usually appear between the ages of 7 and 10 years and fuse with the main body of the epiphysis at puberty. When the secondary centre fuses with the main bone, there may be an unusually large and prominent epiphysis which represents the accessory centre. However, occasionally the extra centre of ossification persists; the junction with the main bone may be fibrous, cartilaginous, or (rarely) a synovial-lined cavity. This ossicle was called the "os subfibulare" by Leimbach (1937) and may be bilateral. The literature contains few references to

J. D. Griffiths, MB BS, Orthopaedic Registrar

M. B. Menelaus, MD, FRCS, FRACS, Chief Orthopaedic Surgeon

The Royal Children's Hospital, Flemington Road, Parkville, Victoria 3052, Australia.

Requests for reprints should be sent to $\mathrm{Mr}$ M. B. Menelaus.

(C) 1987 British Editorial Society of Bone and Joint Surgery $0301-620 \mathrm{X} / 87 / 2042 \$ 2.00$

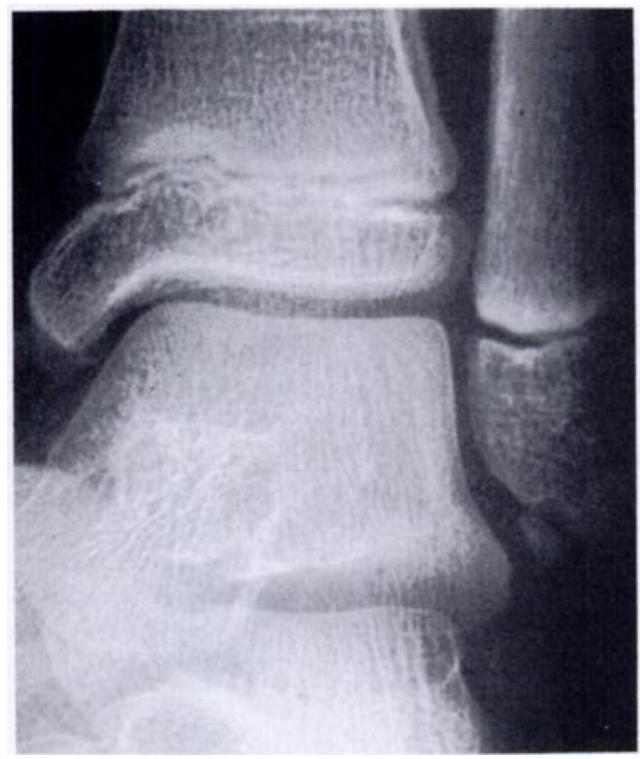

Fig. 1

Case 1. An anteroposterior radiograph showing an accessory ossicle at the distal end of the fibula; there was tenderness precisely at this site. The accessory centre of ossification in the medial malleolus was asymptomatic.

symptomatic ossicles of the lateral malleolus. Bjornson (1956) reported two cases of ankle injury which were treated as fractures; in retrospect, these were considered to be anomalies of ossification with ligamentous injury. There was no prolonged disability in either patient.

In this article, three patients with accessory ossicles of the lateral malleolus are presented; they caused significant and prolonged symptoms which were relieved by excision. 


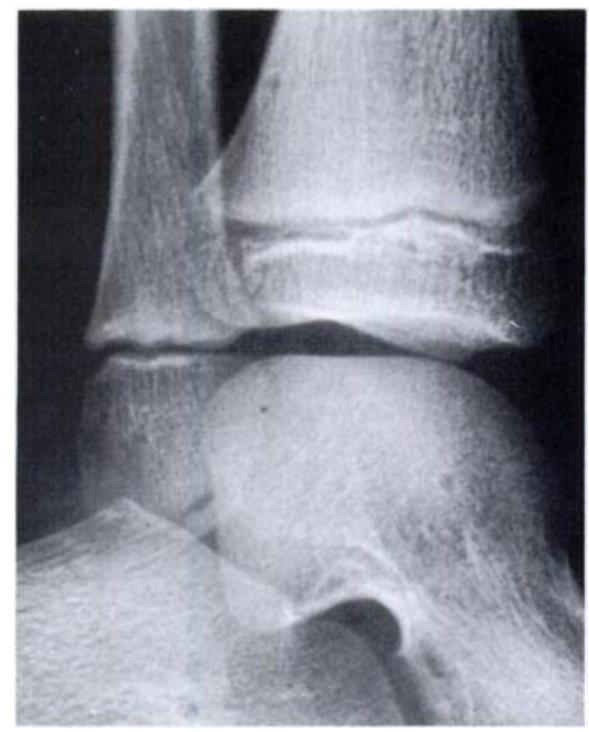

Fig. 2

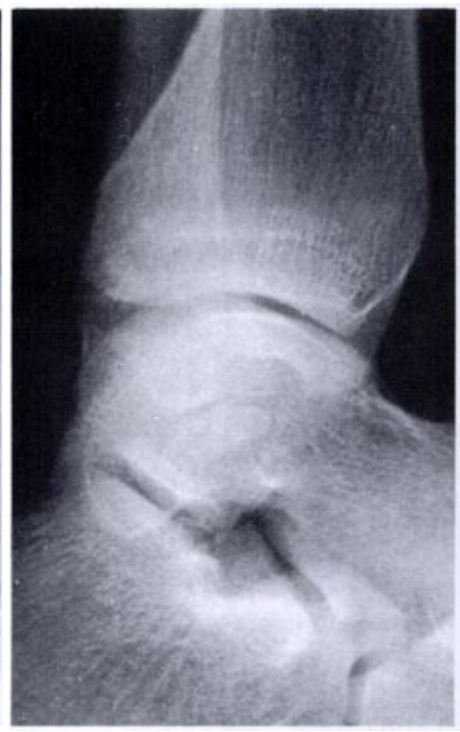

Fig. 3

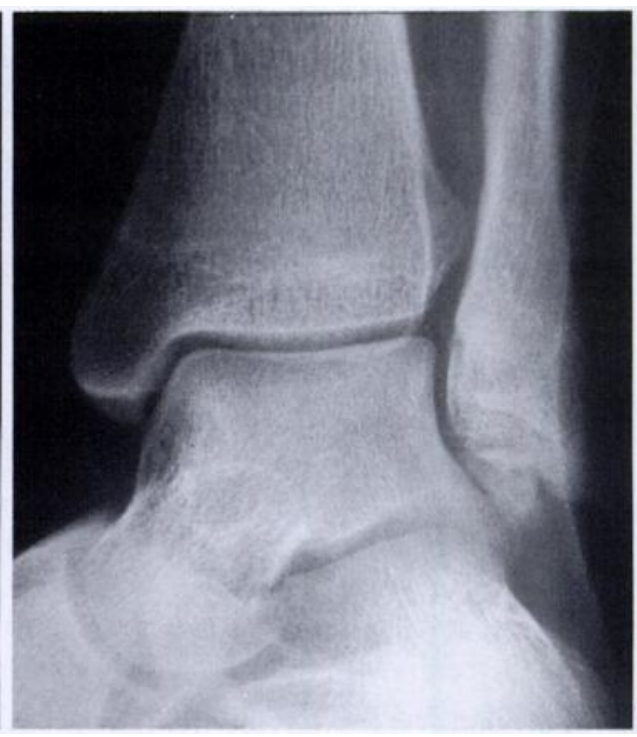

Fig. 4

Case 2. Figure 2 - An oblique radiograph taken at the time of the initial injury when the patient was eight years old. Figures 3 and 4 - Lateral and anteroposterior radiographs of the ankle taken immediately before operation when the patient was 16 years old show that the accessory ossicle has enlarged: note that the lower end of the fibula has a bifid appearance.

\section{CASE REPORTS}

Case 1. A 12-year-old girl presented with a recent ankle injury; on examination she limped and had localised tenderness over the tip of the lateral malleolus. She had twisted her left ankle when 10 years old, and subsequently had had recurrent ankle sprains associated with swelling, pain and a feeling of weakness. Radiographs of her latest injury showed a separate ossicle at the tip of the lateral malleolus (Fig. 1) and a normal contralateral ankle. Two weeks of restricted weight-bearing on crutches was prescribed and her condition improved. Over the next two years, she had intermittent ankle pain which restricted her sporting activity; this was associated with tenderness at the tip of the lateral malleolus noted at each examination.

At the age of 14 years, the ossicle was removed by dissecting it from the lateral ligament and resuturing the ligament to the periosteum. At operation the ossicle was easily recognised by its mobility, that is, it could be rocked medially and laterally on its fibrous attachment to the malleolus. She recovered quickly, her crutches were discarded after three weeks and she soon returned to sport with none of her previous symptoms. Currently, this girl is unrestricted in sport and her only symptom is a mild ache in the ankle after a long run. Radiographs are normal, and there is no laxity of the lateral ligament. Case 2. This 20-year-old man had twisted his left ankle 12 years previously; radiographs taken at the time of his original injury showed a possible fracture of the tip of the lateral malleolus (Fig. 2), and he was treated in plaster for three weeks. On later radiographs, however, there was no evidence of fracture healing. The contralateral ankle was normal. He subsequently had several ankle sprains and intermittent pain over the lateral malleolus which was worse after exercise. Sport was restricted.

At the age of 16, he had persistent symptoms and pinpoint tenderness over an ossicle related to the lateral malleolus. The lateral malleolus had a wide appearance with a bifid distal end and a large accessory ossicle (Figs 3 and 4). The ossicle, which had a wide fibrous attachment to the lateral malleolus, was mobile and was readily excised from the malleolus and from the lateral ligament of the ankle; the ligament was repaired and protected in plaster for six weeks. The patient made a good recovery.

During the next two years, two more ankle sprains occurred, but he was able to continue full sporting activity; since then he has been asymptomatic and plays squash. There is mild laxity of the lateral ligament but radiographs are normal.

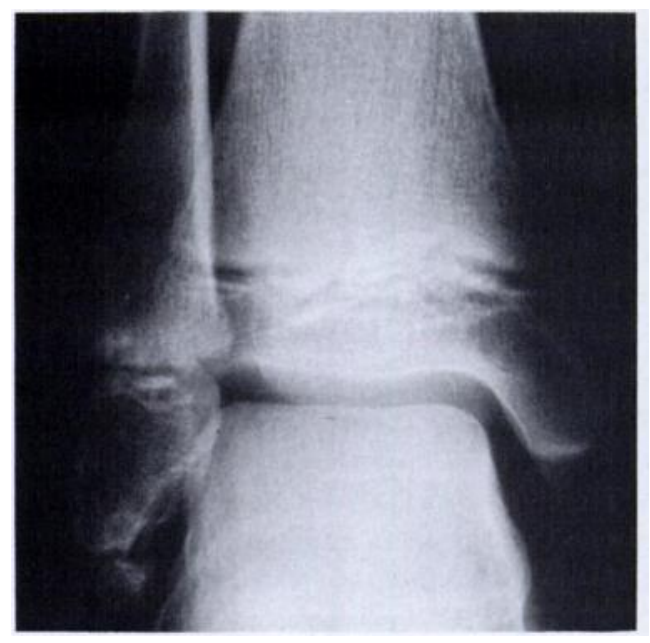

Fig. 5

Case 3. An anteroposterior radiograph showing the accessory ossicle at the tip of the lateral malleolus.

Case 3. This 20-year-old girl had a mild sprain of the right ankle when 10 years old. After that she had had persistent ankle pain associated with tenderness over the tip of the lateral malleolus and recurrent minor sprains. Radiographs showed an accessory ossicle at the tip of the lateral malleolus (Fig. 5) and a normal contralateral ankle.

The ossicle was removed when she was 13 years old because of persistent symptoms and an inability to play sport. A mobile pseudarthrosis was found between the ossicle and the epiphysis of the lateral malleolus. The ossicle was excised and the lateral ligament repaired. Postoperative management was two weeks' non-weightbearing on crutches. She made a good recovery and is currently able to play sport but has aching in the ankle after running. She has mild laxity of the lateral ligament but radiographs are normal. 


\section{DISCUSSION}

These three patients all had similar histories and symptoms: an ankle injury between the ages of 8 to 10 years preceded recurrent ankle sprains for periods ranging from 3 to 8 years, an ossicle was seen on the radiographs at the lower end of the lateral malleolus, and the contralateral ankle was normal. There was also localised tenderness precisely over the lesion which was just anterior to the tip of the lateral malleolus. In all three patients, excision of the fragment and reconstitution of the lateral ligament relieved the symptoms.

The precise cause of symptoms in these patients is conjectural. The most likely explanation is that anomalous ossification centres, not yet fused to the body of the epiphysis, have been subjected to trauma, causing disruption to the fibrous or cartilaginous attachments and resulting in a fibrous union or a pseudarthrosis. Then, mechanical irritation or joint instability may have produced local pain and tenderness as well as recurrent ankle sprains. The operative findings of mobile, separate ossicles attached to the lateral malleolus, and in one case an established pseudarthrosis, were consistent with this suggested pathology.

An alternative theory is that these ossicles result from the non-union of avulsion fractures of the tip of the lateral malleolus. This, however, seems improbable because to produce avulsion fractures of a portion of an epiphysis at other sites requires considerable trauma. No such history of major trauma was given by our patients, all of whom initially suffered only a minor twist or sprain. Furthermore, the ossicles present in our patients were identical in appearance to those found by Powell (1961) in $1 \%$ of normal children in this age group who gave no history of ankle injury. In Case 2, where the radiographs of the original injury were available, there was no progressive callus formation and the gap between the fragment and the epiphysis did not disappear. The ossicle persisted and enlarged in a way typical of an anomalous ossific centre, and the lateral malleolus of this boy had an unusual bifid appearance (Fig. 3); this has been previously described by Bjornson (1956) who attributed the growth alteration to a synchondrosis between the epiphysis and the accessory ossicle. Another possible explanation of this overgrowth might be local increased vascularity from trauma to a pseudarthrosis.

If accessory ossicles are found or suspected on plain radiographs, oblique views, tomograms and comparative views of the other ankle may be needed. Spurious fracture lines may be due to the growth-plate being projected obliquely or to overlap of shadows (Caffey 1967). Accessory ossicles may be unilateral, as in our patients, but are more commonly bilateral (Turek 1984). A knowledge of anatomical variations in children is important when assessing foot and ankle radiographs, and an atlas of these variations is invaluable in a casualty department.

It might be suggested that our three patients were merely suffering from the common form of ankle instability in this age group, which is due to recurrent and incompletely healed injuries of the lateral ligament, and that the accessory ossicle was not relevant to their symptoms. That this was not the case was indicated by the accurately localised tenderness over the ossicle at each examination; the absence of any swelling or tenderness over the lateral ligament or its distal attachment; the finding of a mobile ossicle at operation; and the favourable response to excision of the fragment after 3 to 8 years of disability. Had the symptoms been due to lateral ligament laxity, then the surgery carried out would, if anything, have made the symptoms worse. Furthermore, the age of onset of symptoms in our patients corresponds to the age of appearance of accessory ossicles at the lateral malleolus.

If an accessory ossicle is associated with an ankle injury, it is appropriate to immobilise the ankle in plaster for 3 to 4 weeks, and then to encourage mobilisation and weight-bearing. Excision of the fragment should be reserved for those very few patients with recurrent symptoms over a prolonged period and with tenderness consistently at the site of the ossicle. The rarity of the need for such surgery is indicated by the fact that only three patients required such treatment out of several thousand children with ankle injuries seen over a 26-year period.

\section{REFERENCES}

Bjornson RGB. Developmental anomaly of the lateral malleolus simulating fracture. J Bone Joint Surg [Am] 1956;38-A:128-30.

Caffey J. Pediatric $x$-ray diagnosis. 5th ed. Chicago: Year Book Medical Publishers, 1967.

Francis CC, Werle PP. Appearance of center of ossification from birth to 5 years. Am J Phys Anthropol 1939;24:273-99.

Leimbach G. Beiträge zur Kenntnis der inkonstanten Skeletelamente des Tarsus. (Akzessorische Fusswurzelkaochen) (Untersuchunger an 500 köntgenbildern der Chir. Universitätsklinik zn Jena). Arch Orthop Trauma Surg 1937;38:431-48.

Powell HDW. Extra centre of ossification for the medial malleolus in children: incidence and significance. $J$ Bone Joint Surg $[\mathrm{Br}]$ $1961 ; 43-B: 107-13$.

Shands AR Jr. Accessory bones of foot: $x$-ray study of feet of 1,054 patients. South Med Surg 1931:93:326-34.

Turek SL. Orthopaedics: principles and their application. Vol. 2. 4th ed Philadelphia: JB Lippincott, 1984.

Watkins WW. Anomalous bones of the wrist and foot in relation to injury. JAMA 1937;108:270-3. 